\title{
Relationship between body mass index and mean arterial pressure in normotensive and chronic hypertensive pregnant women: a prospective, longitudinal study
}

Luís Guedes-Martins ${ }^{1,2^{*}}$, Mariana Carvalho ${ }^{3}$, Catarina Silva ${ }^{4}$, Ana Cunha ${ }^{1}$, Joaquim Saraiva ${ }^{1,2}$, Filipe Macedo ${ }^{5}$, Henrique Almeida ${ }^{1,2,6}$ and A. Rita Gaio ${ }^{4,7}$

\begin{abstract}
Background: Being overweight is associated with both higher systolic blood pressure (SBP) and diastolic blood pressure (DBP) during pregnancy and increased risk of gestational hypertensive disorders. The objective of this study was to determine and quantify the effect of body mass index (BMI) on mean arterial pressure (MAP) at several time points throughout pregnancy in normotensive (NT) and chronic hypertensive pregnant (HT) women.
\end{abstract}

Methods: A prospective longitudinal study was carried out in 461 singleton pregnancies (429 low-risk and 32 with chronic arterial hypertension), with measurements taken at the $1^{\text {st }}, 2^{\text {nd }}$, and $3^{\text {rd }}$ trimesters and at delivery. Linear mixed-effects regression models were used to evaluate the time-progression of BMI, SBP, DBP and MAP during pregnancy (NT vs. HT). The longitudinal effect of BMI on MAP, adjusted for the hypertensive status, was investigated by the same methodology.

Results: BMI consistently increased with time in both NT and HT women. In contrast, MAP decreased during the first half of pregnancy, after which it increased until the moment of delivery in both groups. A 5-unit increase in BMl was predicted to produce an increase of approximately $1 \mathrm{mmHg}$ in population MAP values. This effect is independent from the time period and from hypertensive status.

Conclusions: In both NT and HT pregnant women, MAP is strongly (and significantly) influenced by increases in BMI.

Keywords: Pregnancy, Hypertension, Body mass index, Mean arterial pressure

\section{Background}

Chronic arterial hypertension is a serious disorder; if left untreated, it can lead to serious health outcomes, mostly affecting target organs such as the heart, brain, kidney and retina [1].

Women diagnosed with hypertension who become pregnant are at an increased risk for several pregnancy complications, including superimposed preeclampsia [2,3], foetal growth restriction [4], preterm

\footnotetext{
*Correspondence: luis.guedes.martins@gmail.com

'Department of Women and Reproductive Medicine, Hospital Centre of Porto EPE, Largo Prof. Abel Salazar, 4099-001 Porto, Portugal

${ }^{2}$ Department of Experimental Biology, Faculty of Medicine, University of Porto, 4200-319 Porto, Portugal

Full list of author information is available at the end of the article
}

delivery [5], placental abruption [6], and caesarean section $[3,5]$. In addition, because chronic arterial hypertension affects $3-5 \%$ of pregnancies $[7,8]$, it alone is a matter of concern and is increasingly encountered [3]. Obesity is the main risk factor contributing to this increased prevalence; its frequency is increasing among pregnant women, and it is a wellknown risk factor for both adverse maternal [9] and neonatal outcomes $[10,11]$. In fact, increased adiposity during normal pregnancy has been consistently associated with the same medical complications that are associated with chronic arterial hypertension in pregnant women (as described above) [12]. However, the 
mechanisms for these associations are not completely understood.

Several studies have examined the effects of maternal weight on blood pressure levels during different periods of normal pregnancy [10,13-16]. The results suggest that overweight, obesity and morbid obesity are associated with higher systolic (SBP) and diastolic blood pressure (DBP) during pregnancy and increased risks of gestational hypertensive disorders [9].

Nevertheless, studies that have effectively quantified the effects of weight gain on maternal blood pressure during pregnancy are lacking. It has been reasoned that additional data on the effects of increased body mass index (BMI) on mean arterial pressure (MAP) during normal pregnancy would be provided by a parallel study in women with long-term stable essential hypertension, which is a prevalent condition and a known risk factor for serious gestational disorders $[5,7,17]$.

Based on these considerations, this study aimed to determine and quantify the effects of BMI on MAP at several time points throughout pregnancy, in normotensive (NT) and chronic hypertensive (HT) pregnant women.

\section{Methods}

\section{Ethics}

The research protocol was approved by the local ethics committee (IRB protocol number: 133/10 [086-DEFI/126-CES]) of the Centro Hospitalar do Porto - Unidade Maternidade Júlio Dinis (CHP$\mathrm{MJD}$ ), and all of the subjects provided written consent upon receiving an adequate explanation of the study. The methods were carried out in accordance with the approved protocol.

\section{Study population and design}

Between January 2010 and December 2012 a total of 578 pregnant Caucasian women were recruited to participate in the study. According to local pregnancy health policies, the women were referred by their family doctors to the CHP-MJD.

During their first appointment the women were observed by a senior specialist who reviewed their medical history, verified the absence of diabetes and other endocrine disorders, immune diseases, renal diseases, structural heart diseases, haematological conditions and chronic infections; gestational age (GA) was also checked by ultrasonography between 11 and 14 weeks [18].

The inclusion criteria were as follows: (1) singleton pregnancy and gestational age $\leq 14$ weeks, and (2) healthy status or stable chronic arterial hypertension without known target organ involvement.
The exclusion criteria were as follows: (1) patients with multiple gestations, coagulopathy, haematological pathology, diabetes, or any pregnancy-induced hypertension including preeclampsia, and (2) patients who refused to participate. Subjects were also excluded from the study if they had a preterm delivery (birth $<37^{\text {th }}$ gestational week), were lost to follow-up, needed antihypertensive medication, or experienced foetal death.

Chronic arterial hypertension was defined as a blood pressure of $140 / 90 \mathrm{mmHg}$ on more than two occasions before 20 weeks of gestation or after 20 weeks of pregnancy if it persisted beyond 12 weeks postpartum [17].

Before pregnancy, the majority of the hypertensive patients required multiple medications, including thiazide, angiotensin converting enzyme inhibitor, angiotensin receptor blockers, or calcium channel blockers, to control their hypertension. After their first appointment, antihypertensive drugs were discontinued, and their blood pressure was closely monitored. Antihypertensive therapy was restarted if a patient experienced a persistent diastolic pressure between 95 and $99 \mathrm{mmHg}$ or if a systolic pressure $\geq 150 \mathrm{mmHg}$ was observed at any time during their pregnancy. For women with chronic hypertension who enter pregnancy not on antihypertensive treatment, the antihypertensive treatment was initiated when blood pressures were consistently $>160 \mathrm{mmHg}$ systolic and/ or $>105 \mathrm{mmHg}$ diastolic [17].

\section{Definition of time-point measurements}

Anthropometric parameters and blood pressure were measured at the following four time points: 12-14 weeks, 18-22 weeks, 29-33 weeks, and delivery. Each of these time points were converted to a time scale ranging from 0 to 1 . Therefore, the initial time (i.e., before pregnancy) was considered to be time $=0$, and the time of delivery was considered to be time $=1$.

\section{Maternal anthropometrics}

The height $(\mathrm{cm})$ and weight $(\mathrm{kg})$ of each subject were measured without heavy clothing and shoes at each time point. Data about maternal weight just before pregnancy was obtained through questionnaires. Prepregnancy body mass index (BMI) was categorized into the following three categories: lean or normal $\left(16-24 \mathrm{~kg} / \mathrm{m}^{2}\right)$, overweight $\left(25-29 \mathrm{~kg} / \mathrm{m}^{2}\right)$ and obese $\left(30-50 \mathrm{~kg} / \mathrm{m}^{2}\right)$.

\section{Blood pressure assessment}

The blood pressure (BP) was measured using an automated instrument (GE Healthcare Carescape ${ }^{\mathrm{ma}}$ V100 Vital Signs Monitor with DINAMAP Technology, Milwaukee, WI, USA); it was measured two consecutive 
times and averaged. Mean arterial pressure (MAP) was obtained according to the following formula:

$$
M A P=\frac{(2 \times \text { diastolic pressure })+\text { systolic pressure }}{3}
$$

Prior to the measurement, each of the participants was seated and asked to relax for 5-10 min. A cuff (CRITIKON Blood Pressure Cuffs ${ }^{\circ}$, GE Healthcare, $23-33 \mathrm{~cm}$, Milwaukee, WI, USA) was placed around the nondominant upper arm at the level of the heart, with the pressure cuff bladder midline over the brachial artery. A larger cuff $(32-42 \mathrm{~cm})$ was used in patients who had an upper arm that exceeded $33 \mathrm{~cm}$. As enrolment in our study took place during pregnancy, we were unable to measure maternal blood pressure before pregnancy.

\section{Definition of normal pregnancy}

To restrict enrolment to patients with normal course pregnancies, we excluded 117 (20.2 \%) pregnant women who experienced any of the following events during their pregnancies: endocrine disorders, psychiatric disorders, history of bariatric surgery, secondary hypertension, gestational hypertension/preeclampsia, preterm delivery, foetal growth restriction, antihypertensive medication use, multiple gestation, and foetal death. After these exclusions, 461 women who had a normal pregnancy remained. Therefore, the study used basic inclusion criteria, including patients who were healthy or had stable chronic hypertension without known target organ involvement.

\section{Efforts to address potential sources of bias}

Any pregnant women who were seen by our clinical investigator during the study period were considered to be potentially eligible. The pregnancy consultations were randomly scheduled by the hospital administrative staff according to the availability of the clinical investigator (L.G-M.). The patients were consecutively recruited, and the maternal anthropometric and blood pressure measurements were taken by experienced midwives who were aware of the study protocol. All of the pregnancies were supervised by the same physician (L.G-M.), but the inclusion of pregnancies in the study was determined by another researcher who coordinated the review of each clinical case (A.C.).

\section{Statistical analysis}

Univariate analyses included the following standard statistical methods: (1) the chi-square or Fisher's exact tests to compare frequencies from categorical variables or to study the independence between two factors; and (2) t-tests to assess the statistical significance of the difference between the means of two independent populations.
Time was considered a continuous variable, with values $0,0.3,0.5,0.8$ and 1 , in the BMI model [respectively $0.3,0.5,0.8$ and 1 in the systolic blood pressure (SBP), diastolic blood pressure (DBP), MAP, and BMI effect on MAP (MAP-BMI) models]. Whilst 0 and 1 were arbitrarily chosen, the other points were proportional to the observational periods in the study; namely, before pregnancy, 12-14 weeks, 18-22 weeks, 29-33 weeks, and at delivery. Hypertension was a binary variable with normotensive status as the reference category.

Linear mixed-effects (regression) models were used with the observations grouped at the individual level [19]. The random effects in the final models were identified either at the intercept alone, or at the intercept and the time coefficient. In the final models with two random effects, the best structure for the variance-covariance matrix of the random effects was shown to be that of a general positive definite matrix. Whenever the within-group error variance function had to be modelled, the assumption of independence did not seem to be compromised. For the same individual, the errors were assumed to be independent from the random effects; for different individuals, the errors were assumed to be independent. The normality assumptions for the random effects and errors distributions were assessed through graphical analysis; however, for the BMI-time model, the responses had to be logtransformed for the normality of the errors distribution to not be rejected, and the normality assumption within the remaining models was confirmed. Due to sample size constraints, interaction terms were only considered in the MAP-BMI model.

The final models were chosen based on the lowest Bayesian information criterion (BIC) or on the likelihood ratio test, as appropriate. All statistical analyses were carried out using $\mathrm{R}$ version 2.12.1 [20]. The significance level was set at 0.05 .

\section{Results}

Of the 461 women who were enrolled in the study, 429 were normotensive and 32 had chronic arterial hypertension.

Figure 1 shows the number of pregnant women at each stage of the study. The main characteristics and pregnancy outcomes are shown in Table 1 . In $31.9 \%$ of the cases, caesarean section was the mode of delivery; in more than $70 \%$ of those cases, the reason for receiving a caesarean section was prior caesarean delivery, dystocia, foetal distress, or breech presentation (Table 2).

Table 3 describes the maternal anthropometrics and blood pressure during the study period. BMI consistently increased over time in both the NT and HT groups. In contrast, MAP decreased during the first half of pregnancy and then increased until the moment of delivery. 


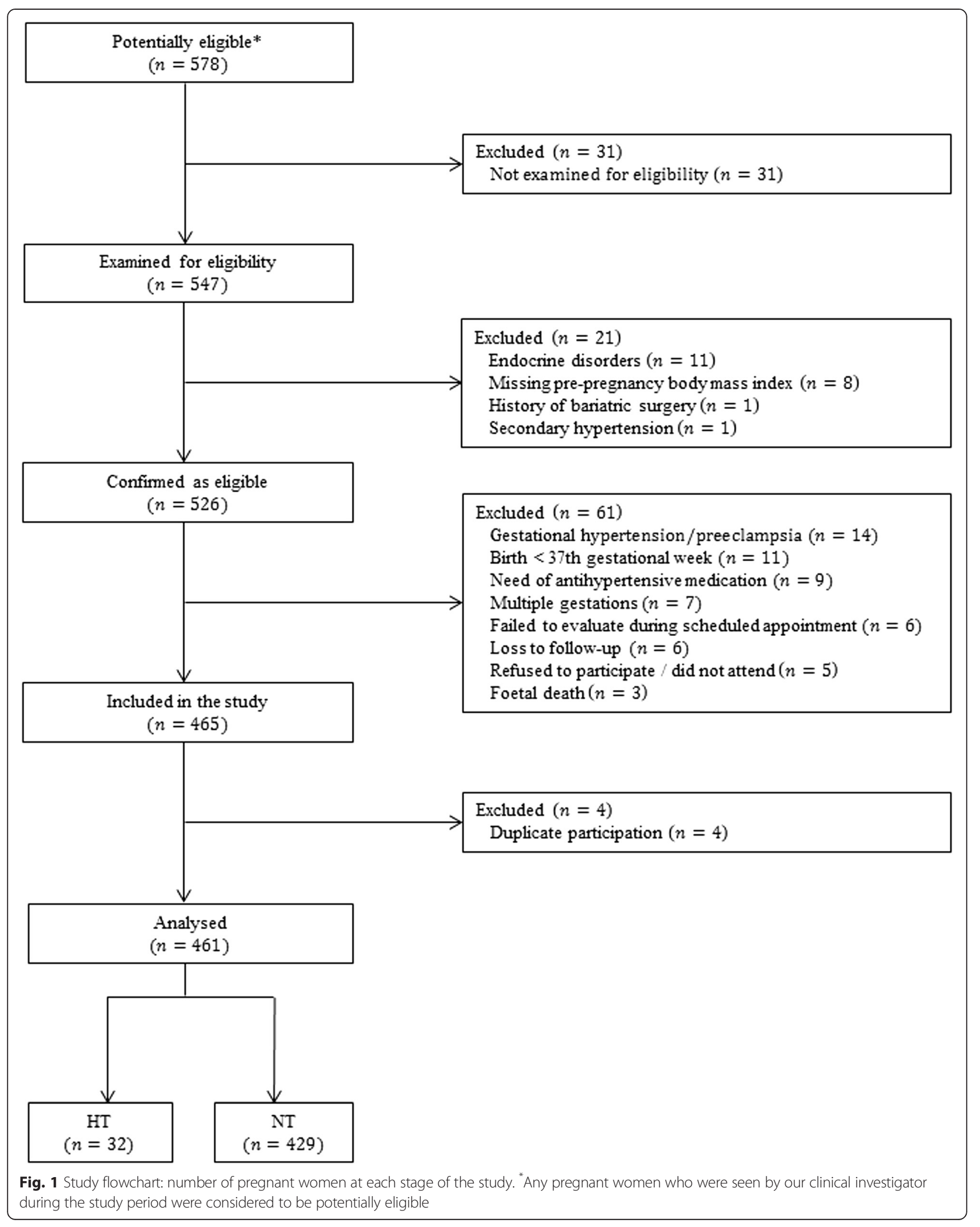


Table 1 Demographic characteristics and pregnancy outcomes of the 461 women included in the analysis

\begin{tabular}{|c|c|c|c|c|c|c|c|c|c|c|}
\hline & & \multirow[b]{2}{*}{$n(\%)$} & \multirow[b]{2}{*}{$p$-value } & \multirow{2}{*}{$\begin{array}{l}\text { Normotensive } \\
n=429\end{array}$} & \multicolumn{2}{|l|}{ Hypertensive } & \multicolumn{3}{|l|}{$\mathrm{BMI}\left(\mathrm{kg} / \mathrm{m}^{2}\right)$} & \multirow[b]{2}{*}{$p$-value $e^{* * *}$} \\
\hline & & & & & $n=32$ & $p$-value ${ }^{* *}$ & $16-24$ & $25-29$ & $30-50$ & \\
\hline \multirow[t]{3}{*}{ Age years [years (\%)] } & $16-24$ & $102(22 \%)$ & $<0.001$ & $102(24 \%)$ & 0 & $<0.001$ & $66(26 \%)$ & $20(17 \%)$ & $16(18 \%)$ & 0.047 \\
\hline & $25-35$ & 303 (66 \%) & & $285(66 \%)$ & $18(56 \%)$ & & $164(65 \%)$ & 78 (66 \%) & $61(67 \%)$ & \\
\hline & $36-43$ & $56(12 \%)$ & & $42(10 \%)$ & $14(44 \%)$ & & 22 (9 \%) & $20(17 \%)$ & $14(15 \%)$ & \\
\hline \multirow[t]{2}{*}{ Parity [n (\%)] } & 0 & $238(52 \%)$ & 0.485 & $226(53 \%)$ & $12(38 \%)$ & 0.140 & $135(54 \%)$ & $54(46 \%)$ & 49 (54 \%) & 0.335 \\
\hline & $\geq 1$ & $223(48 \%)$ & & $203(47 \%)$ & $20(62 \%)$ & & $117(46 \%)$ & $64(54 \%)$ & $42(46 \%)$ & \\
\hline $\begin{array}{l}\text { Gestational age at delivery, } \\
\text { weeks [mean (SD)] }\end{array}$ & $39.22(1.20)$ & - & NA & $39.24(1.17)$ & $38.93(1.68)$ & 0.308 & $39.21(1.18)$ & $39.20(1.28)$ & $39.25(1.22)$ & 0.960 \\
\hline \multirow[t]{2}{*}{ Foetal sex [n (\%)] } & Female & $245(53 \%)$ & 0.177 & $229(53 \%)$ & $16(50 \%)$ & 0.852 & $129(51 \%)$ & $62(53 \%)$ & $54(59 \%)$ & 0.405 \\
\hline & Male & $216(47 \%)$ & & $200(47 \%)$ & $16(50 \%)$ & & $123(49 \%)$ & $56(47 \%)$ & 37 (41 \%) & \\
\hline $\begin{array}{l}\text { Birth weight at delivery, } \\
\mathrm{g} \text { [mean (SD)] }\end{array}$ & $3128(334)$ & - & NA & $3136(329)$ & 3007 (379) & 0.070 & $3116(350)$ & $3167(321)$ & $3108(301)$ & 0.042 \\
\hline \multirow[t]{2}{*}{ Apgar Score Index at $5^{\prime}$} & $<7$ & 0 & NA & $0(0 \%)$ & $0(0 \%)$ & $<0.001$ & 0 & 0 & 0 & $<0.001$ \\
\hline & $7-10$ & $461(100 \%)$ & & $429(100 \%)$ & $32(100 \%)$ & & $252(100 \%)$ & $118(100 \%)$ & 91 (100\%) & \\
\hline
\end{tabular}

BM/ body mass index, $S D$ standard deviation

$\mathrm{p}$ - tested equality of population frequencies amongst the different categories of a variable

* $p$ - tested homogeneity of the proportions between HT (hypertensive) and NT (normotensive)

$\mathrm{p}$ - tested homogeneity of the proportions between normal weight, overweight and obese 
Table 2 Indication for caesarean sections $(n=147)$ in the study sample

\begin{tabular}{|c|c|c|c|c|}
\hline & & \multicolumn{3}{|c|}{ Caesarean deliveries $(\%)^{a}$} \\
\hline & & All (\%) & Normotensive (\%) & Hypertensive (\%) \\
\hline & & $n=147$ & $n=135^{\mathrm{b}}$ & $n=12^{c}$ \\
\hline \multirow[t]{4}{*}{ Primary } & Dystocia & $29(20)$ & $27(20)$ & $2(17)$ \\
\hline & Non-reassuring foetal heart rate & $21(14)$ & $19(14)$ & $2(17)$ \\
\hline & Abnormal presentation & $18(12)$ & $17(13)$ & $1(8)$ \\
\hline & Unsuccessful trial of forceps or vacuum & $14(10)$ & $13(10)$ & $1(8)$ \\
\hline \multirow[t]{3}{*}{ Repeat } & No VBAC attempt & $40(27)$ & $36(27)$ & $4(33)$ \\
\hline & Failed VBAC & $16(11)$ & $15(11)$ & $1(8)$ \\
\hline & Unsuccessful trial of forceps or vacuum & $9(6)$ & $8(6)$ & $1(8)$ \\
\hline
\end{tabular}

${ }^{a}$ Data are shown as absolute (relative, \%) frequencies

The sums of the relative frequencies in the categories were $101 \%^{\mathrm{b}}$ and $99 \%^{\mathrm{c}}$ due to rounding

VBAC vaginal birth after caesarean

Table 3 Description of maternal anthropometrics and blood pressure at each study period

\begin{tabular}{|c|c|c|c|}
\hline & Period & Normotensive & Hypertensive \\
\hline \multirow[t]{5}{*}{ Weight kg, mean (SD) } & Pre-pregnancy & $64.09(12.65)$ & $75.17(17.06)$ \\
\hline & 12-14 weeks & $65.94(13.03)$ & $76.83(17.67)$ \\
\hline & 18-22 weeks & $69.82(13.69)$ & 78.69 (16.96) \\
\hline & 29-33weeks & $75.92(13.99)$ & $85.03(16.34)$ \\
\hline & At delivery & $80.96(14.12)$ & $92.95(16.93)$ \\
\hline \multirow[t]{5}{*}{ BMl kg/m², mean (SD) } & Pre-pregnancy & $25.10(5.18)$ & $28.76(6.54)$ \\
\hline & 12-14 weeks & $25.83(5.38)$ & $29.40(6.79)$ \\
\hline & 18-22 weeks & $27.35(5.66)$ & $30.12(6.52)$ \\
\hline & 29-33weeks & $29.75(5.86)$ & $32.56(6.39)$ \\
\hline & At delivery & $31.73(5.98)$ & 35.59 (6.59) \\
\hline \multirow[t]{5}{*}{ SBP mmHg, mean (SD) } & Pre-pregnancy & - & - \\
\hline & 12-14 weeks & 119.79 (10.62) & $136.22(9.36)$ \\
\hline & 18-22 weeks & $114.37(10.28)$ & $123.65(9.29)$ \\
\hline & 29-33weeks & $119.91(11.03)$ & $140.81(8.52)$ \\
\hline & At delivery & $121.50(11.66)$ & $143.75(8.84)$ \\
\hline \multirow[t]{5}{*}{ DBP mmHg, mean (SD) } & Pre-pregnancy & - & - \\
\hline & 12-14 weeks & 63.05 (8.15) & 76.09 (6.89) \\
\hline & 18-22 weeks & $64.09(11.44)$ & $72.62(6.84)$ \\
\hline & 29-33weeks & $62.93(10.85)$ & $78.94(8.05)$ \\
\hline & At delivery & $66.13(11.54)$ & $76.88(9.21)$ \\
\hline \multirow[t]{5}{*}{ MAP mmHg, mean (SD) } & Pre-pregnancy & - & - \\
\hline & 12-14 weeks & $81.96(6.75)$ & $96.14(6.01)$ \\
\hline & 18-22 weeks & $80.85(8.52)$ & $86.64(4.45)$ \\
\hline & 29-33weeks & $81.93(8.28)$ & $99.56(6.45)$ \\
\hline & At delivery & 84.59 (8.82) & 99.16 (6.91) \\
\hline
\end{tabular}

$B M I$ body mass index, SBP systolic blood pressure, DBP diastolic blood pressure, MAP mean arterial pressure, $S D$ standard deviation

\section{BMI model}

The longitudinal model for BMI estimated a quadratic progression during pregnancy, with the same progression rate for both the NT and HT groups (Fig. 2).

The model for the BMI evolution during pregnancy, for any given rescaled time $t$ and hypertensive status $h$ (equal to 1 for HT and 0 for NT) of the $\mathrm{i}^{\text {th }}$ woman, was as follows:

$$
\log \left(B M I_{i}\right)(t, h)=\beta_{0}+b_{0 i}+\beta_{1} h+\beta_{2} t+\beta_{3} t^{2}+\varepsilon_{i}
$$

where the random effect $b_{0 i}$ was assumed to follow a normal distribution, and the within-group error terms $\varepsilon_{i}$ were assumed to follow a multivariate (5-dimensional) normal distribution with a first-order autoregressive model for their correlation structure. The corresponding estimates are presented in Table 4. The estimates for the correlation parameter $(95 \% \mathrm{CI})$ and the within-group standard error (95\% CI) were $0.943(0.795,0.985)$ and $0.103(0.053$, 0.198), respectively. All fixed effects are multiplicative because the response was log-transformed.

The predicted BMI change during pregnancy consists of two increasing parabolic branches for each hypertensive status that are significantly different from one another; for any given time, the model predicts the hypertensive population to have a mean BMI that is significantly greater than that of the normotensive population.

\section{SBP model}

The model for SBP during pregnancy, for any given rescaled time $t$ and hypertensive status $h$ of the $i^{\text {th }}$ woman, was as follows:

$$
\begin{aligned}
\operatorname{SBP}_{i}(t, h)= & \left(\beta_{0}+b_{0 i}\right)+\beta_{1} h+\left(\beta_{2}+b_{1 i}\right) t \\
& +\beta_{3} t^{2}+\beta_{4} t^{3}+\varepsilon_{i}
\end{aligned}
$$

where the random effects $\left(b_{0 i}, b_{1 i}\right)$ were assumed to follow a bivariate normal distribution with a general positive-definite variance-covariance matrix, and the 

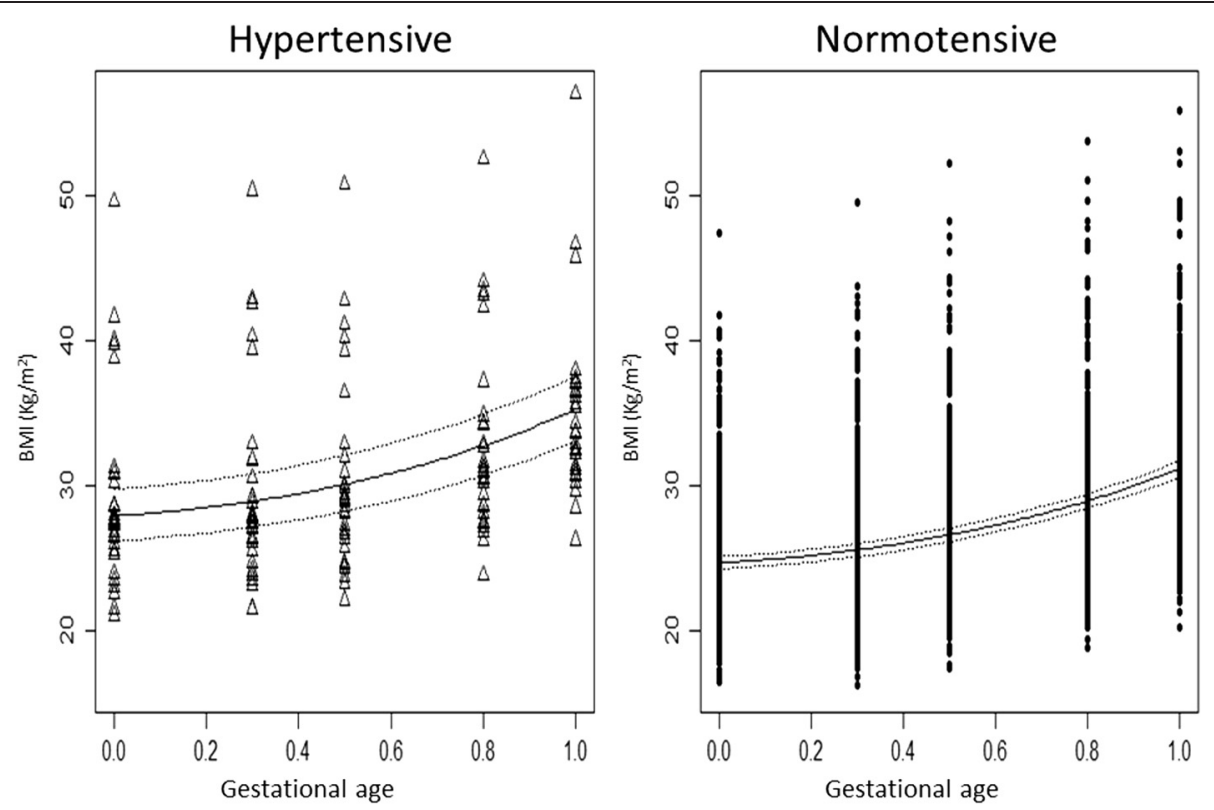

Fig. 2 Expected body mass index (BMI) over time in the hypertensive (triangles) and normotensive (circles) women. The $95 \%$ confidence intervals for the respective predictions are indicated (dashed lines)

within-group error terms $\varepsilon_{i}$ were assumed to follow a multivariate (4-dimensional) normal distribution. The estimates of the effects are presented in Table 4 . The errors were found to have a variance function that is an exponent of the fitted values, with a coefficient $(95 \%$ CI) estimated to be $-0.031(-0.037,-0.025)$. The correlation coefficient $(95 \% \mathrm{CI})$ between the random effects was estimated to be $-0.855(-0.887,-0.815)$.

Two cubic parallel curves were obtained, one for each hypertensive status, and the SBP predictions were significantly higher for the HT group than the NT (Fig. 3). For the normotensive population, the minimum and maximum expected values were $113.5 \mathrm{mmHg}$ and $123.9 \mathrm{mmHg}$, respectively, and they were attained at the (rescaled) time $=0.49$ and time $=0.93$, respectively. For the hypertensive population, the minimum and maximum mean values (attained at the same times) were predicted to be $129.5 \mathrm{mmHg}$ and $139.9 \mathrm{mmHg}$, respectively.

\section{DBP model}

The model for DBP during pregnancy was of a similar form to (2). The obtained estimates for its effects are presented in Table 4. The correlation coefficient (95\% CI) between the random effects was estimated to be -0.831 $(-0.882,-0.760)$. The variance-covariance matrix of the within group errors was diagonal with a variance function that was constant for the hypertensive and normotensive groups. When the error variance within the normotensive population was standardized to 1 , the estimated variance
(95\% CI) of the hypertensive population was 0.691 (0.581, 0.821 ) times the variance prior to standardization.

Two cubic parallel curves were expected, and the DBP values were significantly higher for the HT group than the NT group (Fig. 3). For the normotensive population, the minimum and maximum mean values were predicted to be $63.21 \mathrm{mmHg}$ and $66.38 \mathrm{mmHg}$, respectively, and they were attained at $t=0.64$ and $t=1$, respectively. For the hypertensive population, the minimum and maximum mean values (attained at the same times) were predicted to be $75.16 \mathrm{mmHg}$ and $78.34 \mathrm{mmHg}$, respectively.

\section{MAP model}

The model for MAP during pregnancy was again of a similar form to (2). Its estimates are presented in Table 4. The correlation coefficient $(95 \% \mathrm{CI}$ ) between the two randomeffects was estimated to be $-0.871(-0.828,-0.772)$. The variance-covariance matrix of the within group errors was diagonal with a power variance function based on the fitted values; the variance function coefficient $(95 \% \mathrm{CI})$ was estimated to be $-1.632(-2.206,-1.058)$.

For the MAP model, two cubic parallel curves, one for each hypertensive status, were predicted, and the MAP values were significantly higher in the hypertensive group than the normotensive group (Fig. 4). Between 12-14 weeks, the average MAP values were $82.42 \mathrm{mmHg}$ and $95.43 \mathrm{mmHg}$ in the NT and HT groups, respectively. The values decreased during weeks $18-22$, reaching a minimum of $80.43 \mathrm{mmHg}$ and $93.44 \mathrm{mmHg}$ in the NT and HT groups, respectively. After week 22, the values 
Table 4 Estimates for the fixed and random effects identified by the (longitudinal) mixed-effects model for body mass index (BMI), systolic blood pressure (SBP), diastolic blood pressure (DBP), mean arterial pressure (MAP), and adjusted BMI effect on MAP (MAP-BMI), in normotensive (NT) and chronic hypertensive (HT) women

\begin{tabular}{|c|c|c|c|c|c|c|}
\hline & Fixed effects & Coefficient & Standard error & $p$-value & Random effects & Standard Deviation (95 \% Cl) \\
\hline \multirow[t]{4}{*}{$\mathrm{BMI}$} & Intercept & 3.206 & 0.009 & $<0.001$ & Intercept & $0.158(0.117,0.212)$ \\
\hline & HT (vs. NT) & 0.124 & 0.034 & $<0.001$ & - & - \\
\hline & Time & 0.067 & 0.006 & $<0.001$ & - & - \\
\hline & $(\text { Time })^{2}$ & 0.167 & 0.006 & $<0.001$ & - & - \\
\hline \multirow[t]{5}{*}{ SBP } & Intercept & 183.644 & 3.692 & $<0.001$ & Intercept & $11.237(10.058,12.553)$ \\
\hline & HT (vs. NT) & 16.034 & 1.166 & $<0.001$ & - & - \\
\hline & Time & -346.487 & 20.000 & $<0.001$ & Time & $18.351(16.601,20.285)$ \\
\hline & $(\text { Time })^{2}$ & 539.023 & 32.389 & $<0.001$ & - & - \\
\hline & $(\text { Time })^{3}$ & -253.282 & 16.192 & $<0.001$ & - & - \\
\hline \multirow[t]{5}{*}{ DBP } & Intercept & 57.967 & 3.905 & $<0.001$ & Intercept & $7.036(5.702,8.683)$ \\
\hline & HT (vs. NT) & 11.954 & 0.974 & $<0.001$ & - & - \\
\hline & Time & 32.958 & 21.255 & 0.121 & Time & $15.327(13.516,17.380)$ \\
\hline & $(\text { Time })^{2}$ & -63.105 & 35.012 & 0.072 & - & - \\
\hline & $(\text { Time })^{3}$ & 38.120 & 17.863 & 0.033 & - & - \\
\hline \multirow[t]{5}{*}{ MAP } & Intercept & 96.181 & 2.902 & $<0.001$ & Intercept & $6.719(5.802,7.780)$ \\
\hline & HT (vs. NT) & 13.014 & 0.851 & $<0.001$ & - & - \\
\hline & Time & -74.081 & 15.754 & $<0.001$ & Time & $12.745(11.443,14.196)$ \\
\hline & $(\text { Time })^{2}$ & 107.357 & 25.840 & $<0.001$ & - & - \\
\hline & $(\text { Time })^{3}$ & -44.409 & 13.116 & 0.001 & - & - \\
\hline \multirow[t]{6}{*}{ MAP-BMI } & Intercept & 91.209 & 3.026 & $<0.001$ & Intercept & $6.509(5.604,7.562)$ \\
\hline & HT (vs. NT) & 12.283 & 0.837 & $<0.001$ & - & - \\
\hline & BMI & 0.210 & 0.039 & $<0.001$ & - & - \\
\hline & Time & -75.634 & 15.635 & $<0.001$ & Time & $12.713(11.422,14.150)$ \\
\hline & $(\text { Time })^{2}$ & 108.038 & 25.629 & $<0.001$ & - & - \\
\hline & $(\text { Time })^{3}$ & -45.221 & 13.004 & 0.001 & - & - \\
\hline
\end{tabular}

The NT women were designated as the reference category

increased until delivery, reaching a maximum of $85.05 \mathrm{mmHg}$ and $98.06 \mathrm{mmHg}$, respectively.

\section{Adjusted BMI effect on MAP (MAP-BMI Model)}

The effect of BMI on MAP was obtained by adjusting the previous MAP model for the time-dependent BMI variable. The estimates obtained for the model effects are presented in Table 4. The correlation coefficient (95\% CI) between the random effects was estimated to be -0.832 $(-0.875,-0.776)$. The variance-covariance matrix of the within group errors was diagonal with a variance function that was a power of the fitted values; the variance function coefficient $(95 \% \mathrm{CI})$ was estimated to be $-1.740(-2.300,-1.180)$.

The interaction between hypertensive status and BMI was not statistically significant $(p=0.275)$. Adjusting for BMI led to very similar conclusions regarding the time and the hypertension effects on MAP. With respect to the BMI effect, a 1-unit increase in BMI was predicted to produce an increase of $0.21 \mathrm{mmHg}$ in MAP, or equivalently, a 5-unit increase in BMI was predicted to produce an increase of approximately $1 \mathrm{mmHg}$ in the population MAP values (Fig. 5). This effect was independent of time period and hypertensive status; that is, regardless of the time period and hypertensive status, the predicted BMI effect on MAP remained the same.

Parity was not statistically significant in any of the studied models ( $p=0.443$ for the BMI model; $p=0.712$ for the SBP model; $p=0.471$ for the DBP model; and $p=$ 0.729 for the MAP model).

\section{Discussion}

Most of the normal increase in weight during pregnancy can be attributed to the foetus, breasts, and increases in extravascular fluid, and it causes inevitable demands on the hemodynamic balance of the pregnant. Consequently, blood pressure (BP) and maternal weight measurements play a central role in the 


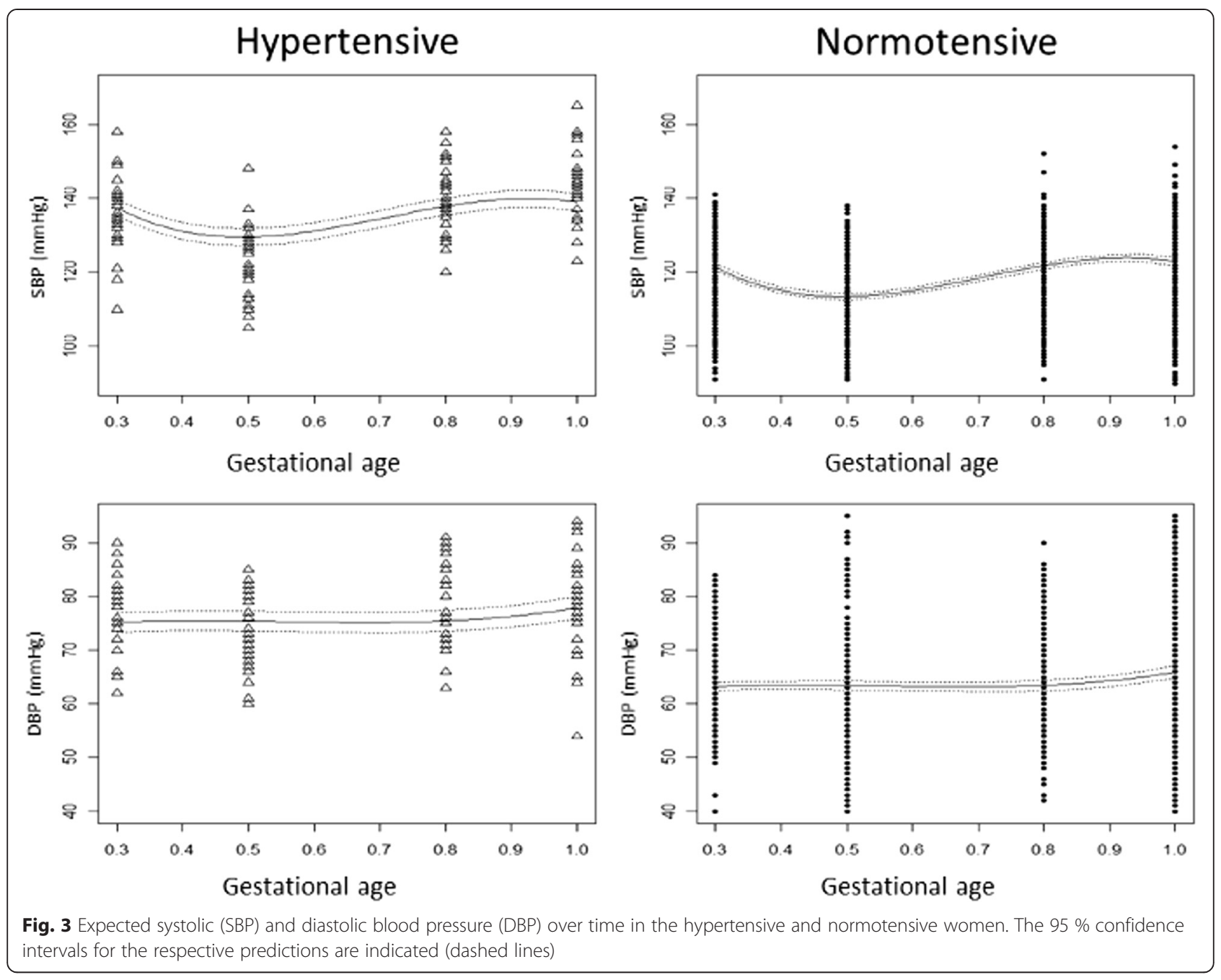

adequate monitoring of pregnancy, during which profound metabolic and circulatory changes occur $[13,15,21,22]$. These cardiovascular changes begin early and include cardiac output increase, blood volume expansion, peripheral vasodilation and blood pressure reduction. Notably, half of the cardiac output increase occurs by 8 weeks of gestation, and cardiac output increases to $30-50 \%(1.8 \mathrm{~L} / \mathrm{min})$ above the typical baseline (i.e., in non-pregnant status) [23-25]. As a consequence, in early pregnancy the uterus receives 3 to $6 \%$ of the cardiac output, whereas at 37-41 weeks it receives approximately 12 $\%$ of the cardiac output [26]. This increase is crucial for an adequate perfusion of the developing fetoplacental unit [27-29].

The results from this prospective study showed that, as in normotensive gestations [30, 31], in chronic hypertensive pregnant women the shapes of the average SBP and MAP trajectories are characterized by a decrease until mid-pregnancy followed by an increase late in pregnancy. This pattern was much less noticeable for DBP, where little and almost no decreases in the average values were observed during pregnancy in the NT and HT groups, respectively. In addition, the differences in the SBP, DBP, and MAP trajectories between the NT and HT groups remained constant throughout pregnancy, and the trajectories temporally progress in a parallel fashion.

To our knowledge, this is the first study to quantify the effects of BMI on MAP in chronic hypertensive pregnant women. We observed that higher BMI values were associated with higher MAP values in all trimesters of pregnancy in both the NT group and the HT group. In addition, regardless of the time period and hypertensive status, the predicted effect of BMI on MAP remained the same; a 5-unit increase in BMI was predicted to produce an increase of approximately $1 \mathrm{mmHg}$ in MAP. 

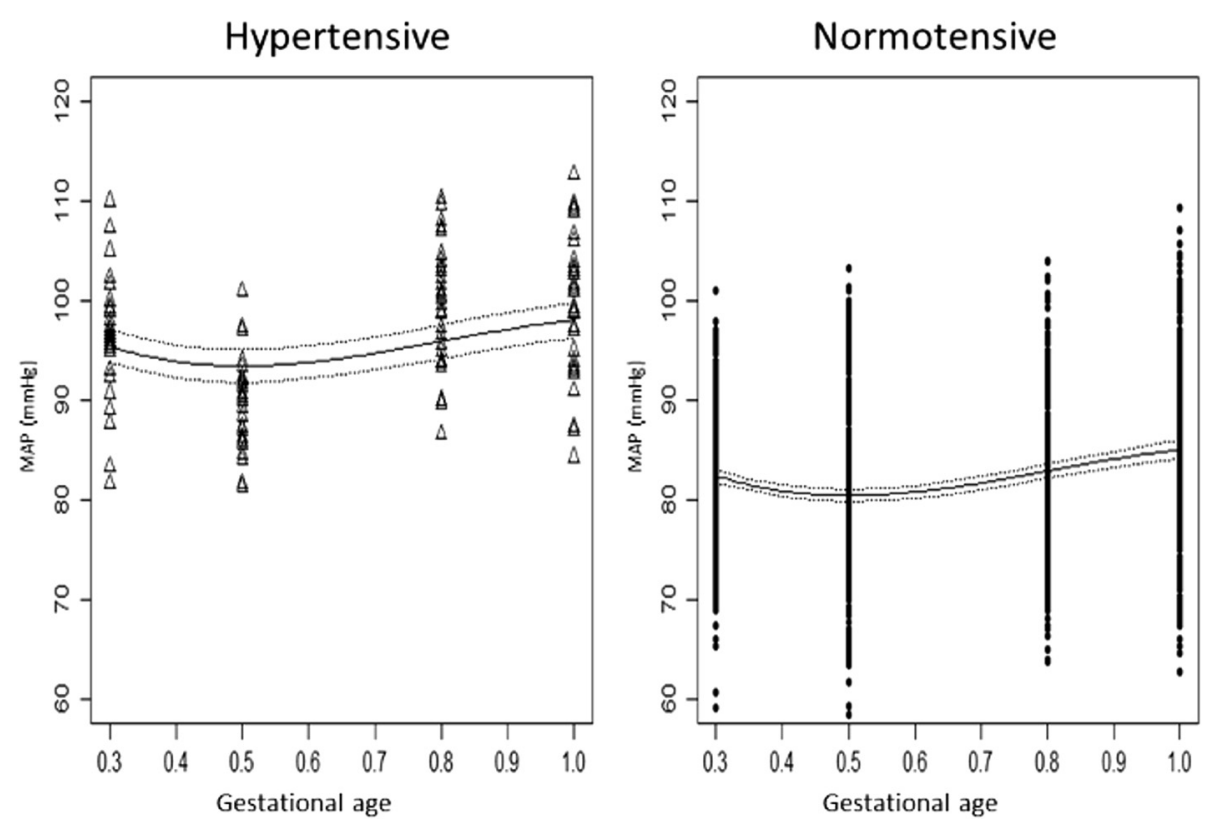

Fig. 4 Expected mean arterial pressure (MAP) over time in the hypertensive (triangles) and normotensive (circles) women. The $95 \%$ confidence intervals for the respective predictions are indicated (dashed lines)

Overall, our findings are in line with the robust association that has been found between increased adiposity and higher blood pressure in humans [32-34]. Particularly, previous studies have verified the blood pressure pattern that occurs during the trimesters of pregnancy in clinically healthy pregnant women [35-37] but not in a population of chronic hypertensive pregnant women.

\section{Statistics and methodological issues}

In all of the models, the two mean curves had a narrow $95 \%$ confidence interval. This suggests that most of the variability was captured by the random effects, which justifies their presence in the models. Moreover, the presence of random effects and the modelling of their variance-covariance structures competed with the correlation structure of the errors, which turned out to have a simple structure. This phenomenon is well-known in the statistical literature on mixed-effects models [19]. The fact that the best variance-covariance structure of the random effects was always a general positive definite matrix is in line with the values of the estimated correlation coefficients and variance estimates that were obtained in the various models.

In the DBP model, the estimate for the time coefficient was not statistically significant; however, this is irrelevant because the estimates of the higher-order terms were all significant.

No interactions between time and the hypertensive status of the women were considered. This was essentially due to the high-order polynomials (third order) that we found for the time variable combined with the sample size of the hypertensive group. Therefore, the predicted mean curves for the hypertensive population turned out to be a translation of the graphical mean values that were predicted for the normotensive population. However, this seemed to only negatively impact the fitting within the hypertensive population in the second evaluation period of the MAP model.

Within the MAP model, the residuals of the hypertensive group were larger than those of the normotensive group. This result was related to the strength of the model, particularly the cubic time progression that ignored the hypertensive status of the women, up to adding a constant. The authors predicted that the presence of interaction terms in the model would improve the model predictions for the hypertensive population. However, this method would be inadequate given the relatively low sample of hypertensive women. Nonetheless, the sample size imbalance between the NT and HT participants is in accordance with the prevalence of chronic hypertension during pregnancy $[7,8]$.

The principal strength of this study was its prospective longitudinal design, which allowed for the assessment of data from the first trimester onwards. In addition, our analyses are based on blood pressure and weight measurements in the clinic (routine); therefore, they reflect the patterns that occur in daily clinical practice as opposed to assessments that are made during trial conditions [15]. 

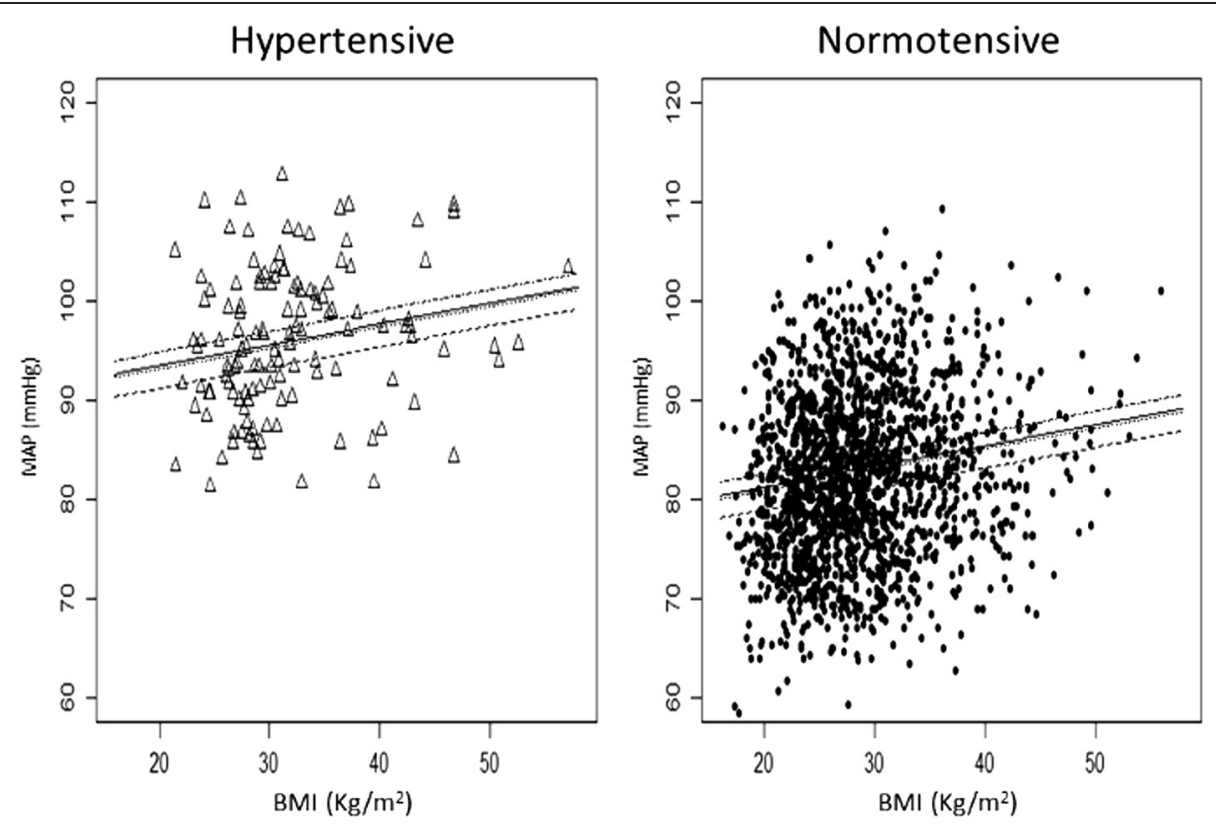

Fig. 5 Expected effects of body mass index on mean arterial pressure, at different time points during pregnancy, in the hypertensive and normotensive pregnant women. Solid line, 12-14 weeks; dashed line, 18-22 weeks; dotted line, 29-33 weeks; dashed-dotted

line, at delivery. The effect is always the same, geometrically reflected on a unique slope, regardless of the time period and hypertensive status

\section{Weight gain and hypertension}

It is thought that weight gain causes hypertension $[38,39]$. In fact, in the general population, many cohort studies indicate that being overweight is a major risk factor for the development of hypertension and that weight loss lowers blood pressure in most hypertensive patients $[1,38]$. This phenomenon has been attributed to increases in weightrelated sympathetic activity, which in turn result in the down-regulation of $\beta$-adrenergic receptors; this downregulation leads to a decreased thermogenic response and, consequently, to an increased propensity for weight gain and adiposity-related insulin resistance [40]. This hypothesis has been strengthened by findings that hypertensive subjects experience a generalized decrease in $\beta$-adrenergic responsiveness, which modulates the development of obesity in hypertension [41, 42]. As a corollary, sympathetic overactivation leads to hypertension and weight gain, and the weight gain further worsens the hypertension $[38,43]$. Additionally, being overweight is a cause of chronic inflammation and oxidative stress, which are involved in the pathophysiology of hypertensive disorders during pregnancy $[44,45]$.

Our results suggest that preventive strategies prior to conception or adequate counselling during pregnancy should be applied to prevent obesity in reproductive-age women and to promote adequate increases in BMI during pregnancy.

\section{Study limitations}

There are several limitations to our study that should be acknowledged. First, the self-reporting of information on many covariates was generally avoided in this study, which limited the availability of detailed information about a large number of potential confounding factors, as certain adverse lifestyle-related determinants of hypertension. Second, information on maternal pre-pregnancy weight was obtained through a questionnaire; thus, it tended to be underestimated and we cannot exclude the possibility of minor misclassification. Third, the study comprised uneventful pregnancies, and thus the results cannot be extrapolated to patients with other forms of hypertensive disease during pregnancy. For the same reasons, we are not able to analyse the effect of BMI gain and the risk of gestational hypertensive disorders or any adverse pregnancy outcomes. Fourth, we were not able to measure MAP changes in relation to the distribution of maternal fat, which is more related to endothelial dysfunction, although BMI is highly correlated with visceral fat [46]. Fifth, the generalizability of our investigation is also limited because our participants were all White women. Sixth, because we did not assess pregnancies with adverse outcomes, the clinical relevance of our findings remains uncertain. 


\section{Conclusions}

This study provides evidence that in normotensive and chronic hypertensive pregnant women, MAP is strongly influenced by increases in BMI starting in the first trimester and lasting until delivery.

\section{Abbreviations}

BIC: Bayesian information criterion; BMI: body mass index; BP: blood pressure; CHP-MJD: Centro Hospitalar do Porto - Unidade Maternidade Júlio Dinis; $\mathrm{Cl}$ : confidence interval; DBP: diastolic blood pressure; GA: gestational age; $\mathrm{HT}$ : chronic arterial hypertension; MAP: mean arterial pressure; NT: normotensive; SBP: systolic blood pressure; SD: standard deviation.

\section{Competing interests}

The authors declare that they have no competing interests.

\section{Authors' contributions}

L.G-M. designed the study, performed data collection, analysed the data, and composed the manuscript. M.C. contributed to data collection, analysed the data, and composed the manuscript; C.S contributed to data collection, and analysed the data; A.C. coordinated the review of clinical cases, and organization of study groups; J.S. designed the study, and analysed the data; F.M. analysed the data, and contributed to the critical revision of the manuscript; H.A. designed the study, and contributed to the critical revision of the manuscript. A.R.G. performed all statistical analyses, and contributed to the critical revision of the manuscript. All authors contributed to the data interpretation and the final version of the manuscript, and they all approved the final manuscript version.

\section{Acknowledgements}

We thank the staff of the Department of Obstetrics of Centro Hospitalar do Porto for their kind contributions to this work. A.R.G. was partially supported by CMUP (UID/MAT/00144/2013), which is funded by FCT (Portugal) with national (MEC) and European structural funds through the FEDER programmes, under the partnership agreement PT2020.

\section{Funding}

None.

\section{Author details}

'Department of Women and Reproductive Medicine, Hospital Centre of Porto EPE, Largo Prof. Abel Salazar, 4099-001 Porto, Portugal. '2Department of Experimental Biology, Faculty of Medicine, University of Porto, 4200-319 Porto, Portugal. ${ }^{3}$ Institute of Biomedical Sciences Abel Salazar, University of Porto, 4050-313 Porto, Portugal. ${ }^{4}$ Department of Mathematics, Faculty of Sciences, University of Porto, 4169-007 Porto, Portugal. ${ }^{5}$ Department of Cardiology, Faculty of Medicine, University of Porto, 4200-319 Porto, Portugal. ${ }^{6}$ Obstetrics-Gynaecology, CUF-Hospital Porto, 4100180 Porto, Portugal.

${ }^{7}$ CMUP-Centre of Mathematics, University of Porto, 4169-007 Porto, Portugal.

Received: 1 July 2015 Accepted: 16 October 2015

Published online: 30 October 2015

\section{References}

1. Messerli FH, Williams B, Ritz E. Essential hypertension. Lancet. 2007;370:591-603.

2. Perni U, Sison C, Sharma V, Helseth G, Hawfield A, Suthanthiran M, et al. Angiogenic factors in superimposed preeclampsia: a longitudinal study of women with chronic hypertension during pregnancy. Hypertension. 2012;59:740-6

3. Seely EW, Ecker J. Chronic hypertension in pregnancy. Circulation. 2014;129:1254-61.

4. Chappell LC, Enye S, Seed P, Briley AL, Poston L, Shennan AH. Adverse perinatal outcomes and risk factors for preeclampsia in women with chronic hypertension: a prospective study. Hypertension. 2008;51:1002-9.

5. Bramham K, Parnell B, Nelson-Piercy C, Seed PT, Poston L, Chappell LC. Chronic hypertension and pregnancy outcomes: systematic review and meta-analysis. BMJ. 2014:348:g2301.

6. Gilbert WM, Young AL, Danielsen B. Pregnancy outcomes in women with chronic hypertension: a population-based study. J Reprod Med. 2007;52:1046-51.
7. Sibai BM. Chronic hypertension in pregnancy. Obstet Gynecol. 2002;100:369-77

8. Lawler J, Osman M, Shelton JA, Yeh J. Population-based analysis of hypertensive disorders in pregnancy. Hypertens Pregnancy. 2007;26:67-76.

9. Gaillard R, Steegers EA, Hofman A, Jaddoe WW. Associations of maternal obesity with blood pressure and the risks of gestational hypertensive disorders. The Generation R Study. J Hypertens. 2011;29:937-44.

10. Crane JM, White J, Murphy P, Burrage L, Hutchens D. The effect of gestational weight gain by body mass index on maternal and neonatal outcomes. J Obstet Gynaecol Can. 2009;31:28-35.

11. Aune D, Saugstad OD, Henriksen T, Tonstad S. Maternal body mass index and the risk of fetal death, stillbirth, and infant death: a systematic review and meta-analysis. JAMA. 2014;311:1536-46.

12. Villamor $E$, Cnattingius $S$. Interpregnancy weight change and risk of adverse pregnancy outcomes: a population-based study. Lancet. 2006;368:1164-70.

13. Miller RS, Thompson ML, Williams MA. Trimester-specific blood pressure levels in relation to maternal pre-pregnancy body mass index. Paediatr Perinat Epidemiol. 2007;21:487-94.

14. Helmreich RJ, Hundley $V$, Varvel P. The effect of obesity on heart rate (heart period) and physiologic parameters during pregnancy. Biol Res Nurs. 2008; 10:63-78.

15. Thompson ML, Williams MA, Miller RS. Modelling the association of blood pressure during pregnancy with gestational age and body mass index. Paediatr Perinat Epidemiol. 2009;23:254-63.

16. Mbah AK, Kornosky JL, Kristensen S, August EM, Alio AP, Marty PJ, et al. Superobesity and risk for early and late pre-eclampsia. BJOG. 2010;117:997-1004.

17. Committee on Hypertension in Pregnancy. Hypertension in pregnancy. Washington, DC: American College of Obstetricians and Gynecologists; 2013.

18. Robinson HP. Sonar measurement of fetal crown-rump length as means of assessing maturity in first trimester of pregnancy. Br Med J. 1973;4:28-31.

19. Pinheiro JC, Bates DM. Mixed-effects models in S and S-PLUS. Springer New York: New York; 2000. p. 4-267.

20. R Development Core Team. R. A Language and Environment for Statistical Computing.R Foundation for Statistical Computing [http://www.r-project.org]. (Accessed: $8^{\text {th }}$ December 2014).

21. Hermida RC, Ayala DE, Iglesias M. Differences in circadian pattern of ambulatory pulse pressure between healthy and complicated pregnancies. Hypertension. 2004:44:316-21.

22. American College of Obstetricians and Gynecologists (ACOG). Task force on hypertension in pregnancy. Hypertension in pregnancy. Report of the American College of Obstetricians and Gynecologists' task force on hypertension in pregnancy. Obstet Gynecol. 2013;122:1122-31.

23. Katz R, Karliner JS, Resnik R. Effects of a natural volume overload state (pregnancy) on left ventricular performance in normal human subjects. Circulation. 1978:58(3 Pt 1):434-41.

24. Capeless EL, Clapp JF. Cardiovascular changes in early phase of pregnancy. Am J Obstet Gynecol. 1989:161(6 Pt 1):1449-53.

25. van Oppen AC, Stigter RH, Bruinse HW. Cardiac output in normal pregnancy: a critical review. Obstet Gynecol. 1996;87:310-8.

26. Flo K, Wilsgaard T, Vårtun A, Acharya G. A longitudinal study of the relationship between maternal cardiac output measured by impedance cardiography and uterine artery blood flow in the second half of pregnancy. BJOG. 2010;117:837-44.

27. Robson SC, Hunter S, Boys RJ, Dunlop W. Serial study of factors influencing changes in cardiac output during human pregnancy. Am J Physiol. 1989;256(4 Pt 2):H1060-5.

28. Guedes-Martins L, Saraiva J, Gaio R, Macedo F, Almeida H. Uterine artery impedance at very early clinical pregnancy. Prenat Diagn. 2014;34:719-25.

29. Guedes-Martins L, Cunha A, Saraiva J, Gaio R, Macedo F, Almeida H. Internal iliac and uterine arteries Doppler ultrasound in the assessment of normotensive and chronic hypertensive pregnant women. Sci Rep. 2014:4:3785

30. Strevens $H$, Wide-Swensson D, Ingemarsson I. Blood pressure during pregnancy in a Swedish population; impact of parity. Acta Obstet Gynecol Scand. 2001;80:824-9.

31. Gaillard R, Bakker R, Willemsen SP, Hofman A, Steegers EA, Jaddoe WW. Blood pressure tracking during pregnancy and the risk of gestational hypertensive disorders: the Generation R Study. Eur Heart J. 2011;32:3088-97.

32. Mokdad AH, Ford ES, Bowman BA, Dietz WH, Vinicor F, Bales VS, et al. Prevalence of obesity, diabetes, and obesity-related health risk factors, 2001. JAMA. 2003;289:76-9. 
33. Paradis G, Lambert M, O'Loughlin J, Lavallée C, Aubin J, Delvin E, et al. Blood pressure and adiposity in children and adolescents. Circulation. 2004;110:1832-8.

34. Gregg EW, Cheng YJ, Cadwell BL, Imperatore G, Williams DE, Flegal KM, et al. Secular trends in cardiovascular disease risk factors according to body mass index in US adults. JAMA. 2005;293:1868-74.

35. Hermida RC, Ayala DE, Mojón A, Fernández JR, Alonso I, Silva I, et al. Blood pressure patterns in normal pregnancy, gestational hypertension, and preeclampsia. Hypertension. 2000;36:149-58.

36. Hermida RC, Ayala DE, Mojón A, Fernández JR. Time-qualified reference values for ambulatory blood pressure monitoring in pregnancy. Hypertension. 2001;38(3 Pt 2):746-52.

37. Ayala DE, Hermida RC. Influence of parity and age on ambulatory monitored blood pressure during pregnancy. Hypertension. 2001;38(3 Pt 2):753-8.

38. Calhoun DA, Grassi G. Weight gain and hypertension: the chicken-egg question revisited. J Hypertens. 2004;22:1869-71.

39. Esler MD, Eikelis N, Lambert E, Straznicky N. Neural mechanisms and management of obesity-related hypertension. Curr Cardiol Rep. 2008;10:456-63.

40. Julius S, Valentini M, Palatini P. Overweight and hypertension: a 2-way street? Hypertension. 2000;35:807-13.

41. Valentini M, Julius S, Palatini P, Brook RD, Bard RL, Bisognano JD, et al. Attenuation of haemodynamic, metabolic and energy expenditure responses to isoproterenol in patients with hypertension. J Hypertens. 2004;22:1999-2006.

42. Masuo K, Katsuya T, Fu Y, Rakugi H, Ogihara T, Tuck ML. Beta2- and beta3adrenergic receptor polymorphisms are related to the onset of weight gain and blood pressure elevation over 5 years. Circulation. 2005;111:3429-34.

43. Newsom SA, Richards JC, Johnson TK, Kuzma JN, Lonac MC, Paxton RJ, et al. Short-term sympathoadrenal inhibition augments the thermogenic response to beta-adrenergic receptor stimulation. J Endocrinol. 2010;206:307-15

44. Guedes-Martins L, Matos L, Soares A, Silva E, Almeida H. AGEs, contributors to placental bed vascular changes leading to preeclampsia. Free Radic Res. 2013;Suppl 1:70-80.

45. Guedes-Martins L, Silva E, Gaio AR, Saraiva J, Soares Al, Afonso J, et al. Fetalmaternal interface impedance parallels local NADPH oxidase related superoxide production. Redox Biol. 2015;5:114-23.

46. Sidney S, Lewis CE, Hill JO, Quesenberry Jr CP, Stamm ER, Scherzinger A, et al. Association of total and central adiposity measures with fasting insulin in a biracial population of young adults with normal glucose tolerance: the CARDIA study. Obes Res. 1999;7:265-72.

\section{Submit your next manuscript to BioMed Central and take full advantage of:}

- Convenient online submission

- Thorough peer review

- No space constraints or color figure charges

- Immediate publication on acceptance

- Inclusion in PubMed, CAS, Scopus and Google Scholar

- Research which is freely available for redistribution 